\title{
An analytical solution of a time-fractional diffusion equation with external force and absorbent term by generalized two-dimensional differential transform method
}

\author{
SANTANU BANERJEE \\ Department of Mathematics \\ Supreme Knowledge Foundation Group of Institutions \\ Hooghly, West Bengal, India \\ santanubandopadhyay@gmail.com
}

\author{
SUDESHNA BANERJEE \\ Department of Mathematics \\ Hooghly Engineering and Technology College \\ Hooghly, West Bengal, India
}

Abstract: In this paper, we have used generalized differential transform method in obtaining a general recurrence relation for determining the solutions of time fractional diffusion equation with external force and absorbent term. Diffusion equations play an improtant part in energy transfer problems. Inclusion of fractional derivatives bring the non-locality aspect into the physical system containing this equation. The obtained relation will help us to solve such equations with various external forces and initial conditions. Three illustrative examples have been discussed.

Key-Words: Time-fractional diffusion equation, External force, Reaction term, Absorbent term, Generalized differential transform method, Mittag-Leffler function

Received: May 31, 2020. Revised: August 26, 2020. Accepted: September 4, 2020. Published: September 9, 2020.

\section{Introduction}

In recent years fractional calculus has been of tremendous use in applied mathematical and engineering problems. Oldham and Spanier [1] was the first to introduce the subject in book level. Later on Miller and Ross [2], Podlubny [3] was instrumental in developing the subject in solving fractional differential equations. Fractional differential equations, mainly diffusion equations were rigorously studied in the nineties [4]-[6]. In the present paper we focus our attention in considering and finding solutions of the following time fractional diffusion equation

$$
\begin{array}{r}
\frac{\partial^{\beta} u(x, t)}{\partial t^{\beta}}=M \frac{\partial^{2} u(x, t)}{\partial x^{2}}-\frac{\partial}{\partial x}(F(x) u(x, t)) \\
-\int_{0}^{t} \lambda(t-\xi) \frac{\partial u(x, t)}{\partial x} d \xi
\end{array}
$$

where $0<\beta \leq 1, M, x, t>0$, with the initial condition

$$
u(x, 0)=f(x)
$$

Here $M$ is the diffusion coefficient, $F(x)$ is the external force, $(t)$ is the time dependent absorbent term which may be related to a reaction diffusion process. Throughout this paper the unknown function $u(x, t)$ is considered to be a causal function of space and time respectively.

There are several analytical methods present for solving diffusion-wave equations of fractional order. Most of these methods are applied in solving time-fractional diffusion equations in presence of external force only. Recently Das has used the variational iteration method (VIM) to find the solution of a time-fractional diffusion equation of order $\beta=\frac{1}{2}$ in [7]. Saha Ray and Bera have used the Adomain decomposition method (ADM) for the same equation in [8]. Das further studied the solutions of time-fractional diffusion equations for arbitrary order $\beta(0<\beta \leq 1$ in [9] and used homotopy perturbation method (HPM) for solving time-fractional diffusion equation with external force and absorbent term in [10].

In this paper the Generalized Differential Transform Method (GDTM) is used to solve the fractional diffusion equation problem in presence of a linear external force and an absorbent term. Using the initial condition, the approximate analytical expressions of $u(x, t)$ for different Brownian motions are obtained. The concept of the differential transform method (DTM) was first proposed by Zhou in [11] while solving some linear and nonlinear electrical circuit problems. The generalized two-dimensional differential transform method was first proposed by Momani et. al. in [12]. The main objective of this paper is to give a general recurrence relation for obtaining the solutions of Eq. (1) with the GDTM. This the first study that this type of problem is solved by GDTM for not only a given external force but also for a time 
dependent absorbent term. We shall show with examples, the effect of the reaction term in Eq. (1) with or without the presence of the linear external force.

\section{Mathematical preliminaries}

There are several versions of fractional derivative of order $\alpha>0$. The two most widely used versions are the Riemann-Liouville and Caputo, where both the definitions use Riemann-Liouville fractional integral and derivatives of whole order. Since in this paper we have to deal with partial derivatives, the definitions need to be presented in the partial differential sense.

Consider a function of two-variables $u(x, y)$. The Riemann-Liouville fractional partial integration of order $\nu$ with respect to $x$ is defined as

$$
D_{x}^{-\nu} u(x, y)=\frac{1}{\Gamma(\nu)} \int_{0}^{x}(x-\xi)^{\nu-1} u(\xi, y) \partial \xi
$$

where $x>0$ and $<\nu \leq 1$, and the Riemann-Liouville fractional partial derivative of order $\alpha$ with respect to $x$ is defined as

$$
D_{x}^{\alpha} u(x, y)=\frac{\partial^{m}}{\partial x^{m}} D_{x}^{-(m-\alpha)} u(x, y)
$$

where $x>0,<\alpha \leq 1$ and $m$ is a positive integer such that $m-1<\alpha \leq m$.

The Caputo fractional partial derivative of order $\alpha$ with respect to $x$ is defined as

$$
D_{x}^{\alpha} u(x, y)=D_{x}^{-(m-\alpha)} \frac{\partial^{m}}{\partial x^{m}} u(x, y)
$$

where $x>0,<\alpha \leq 1$ and $m$ is a positive integer such that $m-1<\alpha \leq m$.

The basic difference between the definitions is that in Caputo's definition we first compute the derivative followed by an integral whereas in RiemannLiouville's definition the computation is reversed. Therefore, in an initial value problem the Caputo fractional derivative operator permits the initial conditions in terms of integer ordered derivatives, but the Riemann-Liouville fractional derivative operator permits the initial conditions in terms of fractional integrals and their derivatives. In case of homogeneous initial condition, these two operators coincide. Throughout this paper, as per our requirement in the problem we shall consider the space and time fractional partial derivatives of the causal function $u(x, t)$ in Caputo sense.

\section{Generalized two-dimensional dif- ferential transform method}

Let us consider an analytic function of two variables $u(x, y)$ and suppose that it can be represented as a product of two continuous single variable functions. The function $u(x, y)$ is presented in terms of a twodimensional infinite power series expansion

$$
u(x, y)=\sum_{k=0}^{\infty} \sum_{h=0}^{\infty} U_{\alpha, \beta}(k, h)\left(x-x_{0}\right)^{k \alpha}\left(y-y_{0}\right)^{h \beta}
$$

where $0<\alpha, \beta \leq 1$ and $U_{\alpha, \beta}(k, h)$ is the generalized differential transform (GDT) of $u(x, y)$ also called the spectrum of $u(x, y)$. It is defined as follows:

$U_{\alpha, \beta}(k, h)=$
$\frac{1}{\Gamma(\alpha k+1) \Gamma(\beta h+1)}\left[\left(D_{x}^{\alpha}\right)^{k}\left(D_{y}^{\beta}\right)^{h} u(x, y)\right]_{\left(x_{0}, y_{0}\right)}$

where $\left(D_{x}^{\alpha}\right)^{k}=D_{x}^{\alpha} D_{x}^{\alpha} . . D_{x}^{\alpha}(k-t i m e s)$.

In usual notation of a function the lower case $u(x, y)$ will represent the original function while the uppercase $U_{\alpha, \beta}(k, h)$ will denote the transformed function. Some useful results of the generalized two-dimensional differential transform method are listed below.

Result 1. If $u(x, y)=v(x, y) \pm w(x, y)$, then $U_{\alpha, \beta}(k, h)=V_{\alpha, \beta}(k, h) \pm W_{\alpha, \beta}(k, h)$.

Result 2. If $u(x, y)=c v(x, y)$, then $U_{\alpha, \beta}(k, h)=c V_{\alpha, \beta}(k, h)$, where $c$ is any constant.

Result 3. If $u(x, y)=v(x, y) w(x, y)$, then $U_{\alpha, \beta}(k, h)=\sum_{r=0}^{k} \sum_{s=0}^{h} V_{\alpha, \beta}(r, h-s) W_{\alpha, \beta}(k-$ $r, s)$.

Result 4. If $u(x, y)=(x-a)^{n \alpha}(y-b)^{m \beta}$, then $U_{\alpha, \beta}(k, h)=\delta(k-n) \delta(h-m)$, where $\delta$ represents the Dirac-delta function.

Result 5. If $f(x)=x^{\lambda} h(x)$, where $\lambda+1>0$ and $h(x)$ has the generalized Taylor's series expansion $h(X)=\sum_{n=0}^{\infty} a_{n}(x-a)^{n \alpha}$ with radius of convergence $R>0,0<\alpha \leq 1$. Then

$$
{ }_{a} D_{x a}^{\gamma} D_{x}^{\beta} f(x)={ }_{a} D_{x}^{\gamma+\beta} f(x)
$$

For all $x \in(0, R)$ if:

(a) $\beta<\lambda+1$ and $\alpha$ arbitrary 
or

(b) $\beta \geq \lambda+1, \gamma$ arbitrary, and $a_{k}=0$ for $k=0,1, . . m-1$, where $m-1<\beta \leq m$.

Result 6. If $u(x, y)=f(x) g(y)$ and $f(x)$ satisfies the conditions imposed in Theorem 5, then

$$
\begin{array}{r}
U_{\alpha, \beta}(k, h)= \\
\frac{1}{\Gamma(\alpha k+1) \Gamma(\beta h+1)}\left[\left(D_{x}^{\alpha k}\right)\left(D_{y}^{\beta h}\right) u(x, y)\right]_{\left(x_{0}, y_{0}\right)}
\end{array}
$$

Result 7(i). If $u(x, y) D_{x}^{\gamma} v(x, y), m-1<\gamma \leq m$ and $v(x, y)=f(x) g(y)$ where $f(x)$ satisfies the conditions in Theorem 5, then

$$
U_{\alpha, \beta}(k, h)=\frac{\Gamma(\alpha k+\gamma+1)}{\Gamma(\alpha k+1)} V_{\alpha, \beta}\left(k+\frac{\gamma}{\alpha}, h\right)
$$

Result 7(ii). If $u(x, y) D_{y}^{\gamma} v(x, y), m-1<\gamma \leq m$ and $v(x, y)=f(x) g(y)$ where $g(x)$ satisfies the conditions in Theorem 5, then

$$
U_{\alpha, \beta}(k, h)=\frac{\Gamma(\beta h+\gamma+1)}{\Gamma(\beta h+1)} V_{\alpha, \beta}\left(k, h+\frac{\gamma}{\beta}\right)
$$

Result 8. If $u(x, y)=D_{x}^{\gamma} D_{y}^{\mu} v(x, y), m-1<\gamma \leq$ $m, n-1<\mu \leq n$ and $v(x, y)=f(x) g(y)$, where the functions $f(x)$ and $g(y)$ satisfy the conditions in Theorem 5, then

$$
\begin{array}{r}
U_{\alpha, \beta}(k, h)= \\
\frac{\Gamma(\alpha k+\gamma+1) \Gamma(\beta h+\mu+1)}{\Gamma(\alpha k+1) \Gamma(\beta h+1)} V_{\alpha, \beta}\left(k+\frac{\gamma}{\alpha}, h+\frac{\mu}{\beta}\right)
\end{array}
$$

The above results were due to some standard deductions in GDTM. Now, we shall present the proof of two important theorems on GDTM, eventually using them in succeeding examples.

Theorem A1. If $u(x, y)=D_{x}^{-\gamma} D_{y}^{\mu} v(x, y)$, $m-1<\gamma \leq m, n-1<\mu \leq n$ and $v(x, y)=f(x) g(y)$, where the functions $f(x)$ and $g(y)$ satisfy the conditions in Theorem 5, then

$$
\begin{gathered}
U_{\alpha, \beta}(k, h)= \\
\frac{\Gamma(\alpha k+\mu+1) \Gamma(\beta h-\gamma+1)}{\Gamma(\alpha k+1) \Gamma(\beta h+1)} V_{\alpha, \beta}\left(k+\frac{\mu}{\alpha}, h-\frac{\gamma}{\beta}\right)
\end{gathered}
$$

where $k+\frac{\mu}{\alpha} \geq 0, k+\frac{\gamma}{\beta} \geq 0$.

Proof. Using the generalized Taylor's expansion of $f(x), g(y)$ and GDT we obtain,

$$
u(x, y
$$

$$
\begin{aligned}
& =D_{x}^{-\gamma} D_{y}^{\mu} v(x, y) \sum_{k=0}^{\infty} \sum_{h=0}^{\infty} V_{\alpha, \beta}(k, h)(x-a)^{k \alpha}(y-b)^{h \beta} \\
& =\sum_{k=0}^{\infty} \sum_{k=0}^{\infty} D_{x}^{-\gamma} D_{y}^{\mu}\left[V_{\alpha, \beta}(k, h)(x-a)^{k \alpha}(y-b)^{h \beta}\right] \\
& =\sum_{k=0}^{\infty} \sum_{h=0}^{\infty} V_{\alpha, \beta}(k, h)(x-a)^{k \alpha-\mu}(y-b)^{h \beta+\gamma} \\
& =\sum_{k=0}^{\infty} \sum_{h=0}^{\infty}\left[\frac{\Gamma(\alpha k+1) \Gamma(\beta h+1)}{\Gamma(\alpha k-\mu+1) \Gamma(\beta h+\gamma+1)}\right. \\
& \left.\quad \times V_{\alpha, \beta}(k, h)(x-a)^{k \alpha-\mu}(y-b)^{h \beta+\gamma}\right] \\
& =\sum_{k=-\frac{\mu}{\alpha}}^{\infty} \sum_{h=\frac{\gamma}{\beta}}^{\infty} \frac{\Gamma(\alpha k+\mu+1) \Gamma(\beta h-\gamma+1)}{\Gamma(\alpha k+1) \Gamma(\beta h+1)} V_{\alpha, \beta}\left(k+\frac{\mu}{\alpha}, h-\frac{\gamma}{\beta}\right)
\end{aligned}
$$

Hence from the definition of GDT we arrive at the proof.

Theorem A2. If the external force $F(x)$ be expanded in Maclaurin's series, $F(x)=\sum_{n=0}^{\infty} a_{n} x^{n}$ with a radius of convergence $R>0$ and the absorbent term be represented as $\lambda(t)=\frac{\eta t^{\gamma-1}}{\Lambda(\gamma)}, 0<\gamma \leq 1$, $\eta>0$, then the GDT of Eq.(1) is

$$
\begin{array}{r}
U_{1, \beta}(k, h+1)= \\
\frac{\Gamma(\beta h+1)}{\Gamma(\beta h+\beta+1)}(k+1)\left[M(k+2) U_{1, \beta}(k+2, h)\right. \\
-\sum_{r=0}^{k+1} a_{r} U_{1, \beta}(k-r+1, h) \\
\left.-\eta \frac{\Gamma(\beta h-\gamma+1)}{\Gamma(\beta h+1)} U_{1, \beta}\left(k+1, h-\frac{\gamma}{\beta}\right)\right]
\end{array}
$$

Proof. Applying GDT to both sides of Eq.(1) and using Theorem A1 we obtain,

$$
\begin{gathered}
\frac{\Gamma(\beta h+\beta+1)}{\Gamma(\beta h+1)} U_{1, \beta}(k, h+1)= \\
M(k+1)(k+2) U_{1, \beta}(k+2, h) \\
-(k+1) \sum_{r=0}^{k+1} \sum_{s=0}^{h} \sum_{n=0}^{\infty} a_{n} \delta(r-n) \delta(h-s) U_{1, \beta}(k-r+1, s) \\
-\eta(k+1) \frac{\Gamma(\beta h-\gamma+1)}{\Gamma(\beta h+1)} U_{1, \beta}\left(k+1, h-\frac{\gamma}{\beta}\right)
\end{gathered}
$$

Considering the definition of the Dirac- function we obtain the result. 
Furthermore, we can easily see that the GDT of 2) i.e. of the initial condition $u(x, 0)=f(x)$ is

$$
U_{1, \beta}(k, 0)=\frac{1}{k !} \frac{d^{k} f}{d x^{k}}
$$

\section{Numerical examples}

Example 1. Taking $F(x)=-x, \lambda=0, M=1$, $f(x)=x$ i.e. in presence of only external force, we get the following initial value problem:

$$
\begin{array}{r}
D_{t}^{\beta} u=D_{x}^{2} u+D_{x}^{1}(x u) \\
u(x, 0)=x
\end{array}
$$

Since $F(x)=-x$, we have $a_{n}=\left\{\begin{array}{cc}-1, & n=1 \\ 0, & n \neq 1\end{array}\right.$ and substituting $f(x)=x$ in Eq.(9), we get

$$
U_{1, \beta}(k, 0)= \begin{cases}1, & k=1 \\ 0, & k \neq 1\end{cases}
$$

Then using the general recurrence relation (8), we get

$$
U_{1, \beta}(k, h)=\left\{\begin{array}{cc}
\frac{2^{h}}{\Gamma(h \beta+1)}, & k=1 \\
0, & k \neq 1
\end{array}\right.
$$

Hence, the solution of (10) is given by

$$
u(x, t)=x E_{\beta}\left(2 t^{\beta}\right)
$$

where $E_{p}=\sum_{i}=0^{\infty} \frac{t^{i}}{\Gamma(p i+1)}, p>0$ is the MittagLeffler function in one parameter.

The same result was been obtained by Das et al. [10]. Also if we consider $\beta=\frac{1}{2}$, the solution is in complete agreement with that obtained by Saha et al. [8] and Das [7].

Example 2. Taking $\beta=\frac{1}{2}, F(x)=-x, \lambda=1$, $M=1, f(x)=x$ i.e. in presence of both linear external force and absorbent term, we get the following initial value problem:

$$
\begin{array}{r}
D_{t}^{\frac{1}{2}} u=D_{x}^{2} u+D_{x}^{1}(x u)-D_{t}^{-1} D_{x}^{1} u \\
u(x, 0)=x
\end{array}
$$

Since $F(x)=-x$, we have $a_{n}=\left\{\begin{array}{cc}-1, & n=1 \\ 0, & n \neq 1\end{array}\right.$ and substituting $f(x)=x$ in Eq.(9), we get

$$
U_{1, \frac{1}{2}}(k, 0)= \begin{cases}1, & k=1 \\ 0, & k \neq 1\end{cases}
$$

Then using the general recurrence relation (5), we get

$$
U_{1, \frac{1}{2}}(k, h)=\left\{\begin{array}{cc}
\frac{2^{h-2}-1}{\Gamma\left(\frac{h}{2}+1\right)}, & k=0 \\
\frac{2^{h}-1}{\Gamma\left(\frac{h}{2}+1\right)}, & k=1 \\
0, & k \neq 0,1
\end{array}\right.
$$

Hence, the solution of (11) is given by

$$
u(x, t)=x E_{\frac{1}{2}}\left(2 t^{\frac{1}{2}}\right)-t^{\frac{1}{2}} E_{\frac{1}{2}}\left(K t^{\frac{1}{2}}\right)
$$

where $K^{r}=\left(2^{r}-1\right), r \in \mathbb{Z}^{+} \cup\{0\}$.

Example 3. Taking $\beta=\frac{1}{2}, F(x)=0, \lambda=1$, $M=0, f(x)=x$ i.e. in presence only the absorbent term, we get the following initial value problem:

$$
\begin{array}{r}
D_{t}^{\frac{1}{2}} u=D_{t}^{-1} D_{x}^{1} u \\
u(x, 0)=x
\end{array}
$$

Using similar calculations as the above two examples we get the solution of (12),

$$
u(x, t)=x-t
$$

\section{Conclusion}

In this article we have presented a general recurrence relation for Eq.(1) along with the initial condition (2) using GDTM. The usage of this method is more simple and concise when compared to other existing methods such as ADM, HPM, etc. The effect of the reaction term on time-fractional diffusion equation with or without the presence of external force has been analyzed.

\section{References:}

[1] K.B.Oldham, J.Spanier, The Fractional Calculus-Theory and application of differentiation and integration to arbitrary order, Academic Press, Inc. 1974. 299-309.

[2] K.S.Miller, B.Ross, An introduction to the fractional calculus and fractional differential equations, John Wiley Sons, Inc. 1993.

[3] I. Podlubny, Fractional differential equations, Academic Press. 1999.

[4] J.M.Angulo, M.D.Ruiz-Medina, V.V.Anh, W.Grecksch, , Fractional diffusion and fractional heat equation, Adv. Appl. Probab. 32, 2000, pp. 1077-1099.

[5] A.N. Kochubei, Diffusion of fractional order , Differ. Uravn. 26, 1990, pp. 485-492. 
[6] F. Mainardi, The fundamental solutions for the fractional diffusion-wave equation, Appl. Math. Lett. 9, 1996, pp. 23-28.

[7] S. Das, Analytical solution of a fractional diffusion equation by variational iteration method, Comput. Math. Appl. 57, 2009, pp. 483-487.

[8] S.S. Ray, R.K. Bera, Analytical solution of a fractional diffusion equation by Adomian decomposition method, Appl. Math. Comput. 174, 2006, pp. 329-336.

[9] S. Das, A note on fractional diffusion equations, Chaos Solit. Fract. 42, 2009, pp. 2074-2079.

[10] S. Das, R. Kumar, P.K. Gupta, Approximate analytical solutions for Fractional space- and time- partial differential equations using homotopy analysis method, Appl. App. Math. 5, 2010, pp. 1641-1659.

[11] J.K. Zhou, Differential Transformation and its Applications for Electrical Circuits, Huazhong Univ. Press, Wuhan, China, 1986.

[12] S. Momani, Z. Odibat, V.S. Erturk, On undamped heat waves in an elastic solid, Phy. Lett. A 370, 2007, pp. 379-387.

\section{Creative Commons Attribution License 4.0 (Attribution 4.0 International, CC BY 4.0)}

This article is published under the terms of the Creative Commons Attribution License 4.0

https://creativecommons.org/licenses/by/4.0/deed.en US 